\title{
Nutritional quality and food expenditure in preschool children
}

\author{
Qualidade nutricional e gastos com a alimentação de pré-escolares
}

\author{
Fernanda Rauber' ${ }^{1}$, Márcia R. Vitolo²
}

\section{Resumo}

Objetivo: Avaliar a correlação dos gastos com a alimentação e a qualidade dietética de pré-escolares de baixa condição socioeconômica, considerando a ingestão de micronutrientes e alimentos de alta densidade de açúcar e gordura.

Métodos: Estudo transversal realizado com 346 crianças de 3 a 4 anos de idade que participaram do estudo "Dez passos em ação I" (BRATSA I), uma coorte aninhada a um ensaio de campo randomizado. Foram realizados dois inquéritos recordatórios de 24 horas. Calculou-se o gasto com a alimentação utilizando o preço dos alimentos coletado em diferentes estabelecimentos e ajustado para as quantidades ingeridas.

Resultados: A média de gasto com a alimentação das crianças estimada para um mês foi de $R \$ 100,17 \pm 34,1$. A ingestão de ferro ( $r=$ $0,115 ; p=0,033)$, zinco $(r=0,214 ; p<0,001)$ e vitaminas $A(r=$ $0,197 ; p<0,001)$ e $C(r=0,162 ; p<0,001)$, ajustada para 1.000 kcal, mostrou correlação positiva com os gastos com alimentação $/ 1.000 \mathrm{kcal}$. Não houve diferença significativa nos gastos com alimentação/1.000 kcal em relação ao risco para excesso de peso $(p=0,208)$ e ao consumo de alimentos de alta concentração de açúcar e gordura $(p=0,894$ e $p=0,964)$.

Conclusões: $O$ estudo mostrou que o consumo de energia fornecido por alimentos ricos em açúcar e gordura não está associado com os gastos com a alimentação de crianças de 3 a 4 anos; por outro lado, a qualidade nutricional, avaliada pela ingestão de micronutrientes essenciais, mostrou correlação positiva com os gastos com a alimentação.

J Pediatr (Rio J). 2009;85(6):536-540: Custos e análise de custo, alimentação, nutrição da criança, micronutrientes.

\begin{abstract}
Objective: To assess correlations between the cost and the nutritional quality of the diets of preschool children from low socioeconomic status families, taking into account intakes of micronutrients and foods with high concentrations of sugars and fats.
\end{abstract}

Methods: This was a cross-sectional study undertaken with 346 children aged 3 to 4 years recruited for the "Ten steps in action" (BRATSA I) study, who comprise a nested cohort from the randomized field study. Two 24-hour dietary recall surveys were conducted. Expenditure on food was calculated by taking the price of each food, as verified at a number of different establishments, and adjusting it for the quantity eaten.

Results: Mean expenditure on food for one child was $R \$ 100.17 \pm 34.1$ per month. There was a positive correlation between intakes of iron $(r=$ $0.115 ; p=0.033)$, zinc $(r=0.214 ; p<0.001)$, and vitamins $A(r=0.197$; $p<0.001)$ and $C(r=0.162 ; p<0.001)$, adjusted to $1,000 \mathrm{kcal}$, and expenditure on food $/ 1,000 \mathrm{kcal}$. There were no significant relationships between expenditure on food $/ 1,000 \mathrm{kcal}$ and risk of overweight $(p=$ $0.208)$ or intake of foods with a high fat or sugar content $(p=0.894$ and $\mathrm{p}=0.964$ ).

Conclusions: The study found that consumption of energy provided by fat and sugar-rich foods was not associated with expenditure on feeding these 3-to-4-year-olds. In contrast, nutritional quality, assessed in the form of essential micronutrient intakes, demonstrated a positive correlation with food costs.

J Pediatr (Rio J). 2009; 85(6):536-540: Costs and cost analysis, child nutrition, micronutrients.

de açúcar refinado e redução do consumo de carboidratos complexos. Além disso, a pesquisa mostrou estagnação ou redução do consumo de leguminosas, verduras, legumes, frutas e sucos naturais pela população, atingindo o consumo desses alimentos apenas um terço das recomendações ${ }^{1}$. Este padrão alimentar, considerado como fator de risco para

1. Mestranda, Ciências da Saúde, Universidade Federal de Ciências da Saúde de Porto Alegre (UFCSPA), Porto Alegre, RS.

2. Doutora, Ciências Biológicas, Universidade Federal de São Paulo (UNIFESP), São Paulo, SP.

Não foram declarados conflitos de interesse associados à publicação deste artigo.

Apoio financeiro: Conselho Nacional de Desenvolvimento Científico e Tecnológico (CNPq).

Como citar este artigo: Rauber F, Vitolo MR. Nutritional quality and food expenditure in preschool children. J Pediatr (Rio J). 2009;85(6):536-540.

Artigo submetido em 09.07.09, aceito em 30.09.09.

doi:10.2223/JPED.1955 
deficiências nutricionais e excesso de peso, tende a ser consumido por populações de baixa condição socioeconômica e recursos limitados ${ }^{2}$.

A qualidade da alimentação tem sido relacionada com os gastos com a alimentação ${ }^{3,4}$. Estudos demonstraram que maiores gastos com a alimentação estão associados ao baixo teor de energia da dieta, a um menor percentual de gordura total e saturada e a uma maior ingestão de vitaminas, supondo, a partir dessa demonstração, que uma alta qualidade dietética implica maiores gastos em populações de baixa renda 5,6 .

Não há estudos no Brasil que tenham avaliado os gastos e o valor nutricional da alimentação de crianças de baixo nível socioeconômico. Dessa forma, objetivou-se avaliar se os gastos com a alimentação estão correlacionados com a qualidade nutricional da dieta, considerando a ingestão de micronutrientes, e com o consumo de alimentos com alta densidade de açúcar e gordura entre crianças de baixo nível socioeconômico.

\section{Métodos}

Estudo transversal dos dados coletados no estudo "Dez passos em ação I" (BRATSA I), uma coorte aninhada a um ensaio de campo randomizado realizada entre outubro de 2001 e junho de 2002 para a implementação dos "Dez passos da alimentação saudável: guia alimentar para crianças menores de dois anos"7. As crianças que participaram do estudo foram recrutadas no Hospital Centenário, na cidade de São Leopoldo (RS). Estudantes de graduação em nutrição foram capacitados para realizar a coleta de dados antropométricos, alimentares, sociodemográficos e de condições de saúde da criança por meio de visitas domiciliares realizadas aos 6 e 12 meses de idade. Considerando poder de $90 \%$ e nível de significância de $5 \%$, o cálculo do tamanho da amostra para as análises dos gastos com a alimentação foi de 124 crianças.

Entre novembro de 2005 e maio de 2006, essas mesmas crianças com idades entre 3 e 4 anos foram localizadas em seus domicílios. A coleta de dados sociodemográficos e dietéticos e a realização das medidas antropométricas (peso e altura) foram novamente realizadas por estudantes de nutrição treinados. A confirmação dos dados coletados foi realizada em $5 \%$ da amostra por telefone. Foi agendada a avaliação ambulatorial em uma unidade de saúde municipal e realizada nova avaliação dietética.

Para a realização das medidas antropométricas, a criança foi pesada descalça e vestindo roupas leves em balança digital (Filizola ${ }^{\circledR}$ ) com variação de $100 \mathrm{~g}$. A estatura foi obtida utilizando um estadiômetro (Seca ${ }^{\circledR}$ ) fixado em uma parede lisa, com a criança em posição ereta e com os calcanhares encostados na parede. A classificação do estado nutricional foi realizada por meio do programa Anthro da Organização Mundial de Saúde (OMS) ${ }^{8}$, utilizando como critério para risco de excesso de peso valores superiores a 1 escore $z$ (um desvio padrão).

Os dados dietéticos foram obtidos por meio de dois inquéritos recordatórios de 24 horas realizados com intervalo entre 15 e 30 dias. O primeiro foi realizado no domicílio e o outro no momento da avaliação ambulatorial. As mães foram questionadas sobre todos os alimentos e bebidas consumidos pela criança no dia anterior. Os entrevistadores questionavam com detalhe os tipos de alimentos, quantidades, marcas e métodos de preparação. Uma revisão detalhada de todos os alimentos relatados era realizada ao final da entrevista para a correção de algum dado errôneo ou esquecido. As porções consumidas pelas crianças foram observadas com o auxílio de um álbum de fotos de utensílios e alimentos elaborados especialmente para a pesquisa, obtidas através de medidas caseiras (xícara, colher de sopa, colher de chá). O cálculo nutricional da ingestão alimentar foi realizado utilizando o programa NutWin, versão 1.5 , ampliado com a adição de alimentos disponíveis em tabelas de composição química de alimentos 9,10 e/ou fornecidos pelas indústrias brasileiras.

Para avaliar a ingestão de alimentos de alta densidade de açúcar (ADA) e alta densidade de gordura (ADG), os alimentos presentes nos inquéritos recordatórios 24 horas foram classificados em ADA quando tinham $50 \%$ ou mais de açúcar por $100 \mathrm{~g}$ na composição (refrigerante, gelatina, bala e suco artificial) e em ADG quando tinham $30 \%$ ou mais de gordura por $100 \mathrm{~g}$ (salgadinho, bolacha recheada, embutidos, frituras e chocolate). A média de ingestão dos dois recordatórios foi utilizada para as análises. Considerando as recomendações de ingestão de açúcar e gordura saturada para crianças de 3 e 4 anos, $10 \%$ das calorias totais ${ }^{11}$, utilizou-se o ponto de corte de $150 \mathrm{kcal}$ provindas desses alimentos para classificar como alto o consumo de ADA e de ADG. A ingestão de ferro, zinco e vitaminas $A$ e $C$ de cada criança foi calculada com o objetivo de verificar a qualidade da dieta das crianças. Para as análises, utilizou-se a média de ingestão dos dois inquéritos recordatórios 24 horas ajustada para $1.000 \mathrm{kcal}$.

Todos os alimentos presentes nos recordatórios 24 horas foram colocados em uma planilha específica para facilitar a busca dos preços. A partir desta, foi coletado o preço de cada alimento de três marcas diferentes em um estabelecimento grande (super ou hipermercado) e um pequeno (mercado, minimercado, padaria). Não houve critério para a seleção dos estabelecimentos. No caso de algum item alimentar faltante, ou quando o número de pelo menos três marcas diferentes de um alimento não era atingido, outros locais semelhantes eram pesquisados, sendo os estabelecimentos pequenos aqueles com maiores problemas devido ao número restrito de alimentos e marcas. Em relação às frutas, verduras e legumes, por exemplo, coletou-se o preço dos tipos mais consumidos pela população do estudo em seis estabelecimentos diferentes, três grandes e três pequenos, assim como no caso de produtos com marcas específicas. A partir dos preços obtidos, realizou-se a média do custo de cada alimento por $100 \mathrm{~g}$. Obteve-se o preço de 104 produtos de acordo com os critérios acima descritos.

O preço de sete alimentos, caracterizados por serem lanches rápidos, foi coletado de estabelecimentos não fixos. Em relação às preparações, o cálculo do custo foi realizado por meio de fichas técnicas elaboradas no laboratório de Dietética da Universidade Federal de Ciências da Saúde de Porto Alegre (UFCSPA), Porto Alegre (RS). Das 56 preparações elaboradas, a maior parte constituiu-se de bolos e biscoitos 
caseiros, purês, panquecas, lasanhas, tortas e diferentes sobremesas. Obteve-se o custo por $100 \mathrm{~g}$ de cada preparação. Além disso, para realizar o cálculo dos recordatórios dietéticos foi elaborada uma planilha com o fator de correção e de rendimento dos alimentos que sofrem alteração de peso após o processo de cocção. A partir da obtenção destes dados, os gastos alimentares de cada recordatório dietético foram calculados. Para facilitar a análise dos dados, estimou-se o gasto mensal (referente a 30 dias), utilizando-se a média dos dois recordatórios de cada criança ajustada para 1.000 kcal para evitar possíveis fatores de confusão.

O teste de Kolmogorov foi utilizado para verificar a assimetria dos dados, e os testes $t$ de Student e ANOVA para analisar a associação entre as variáveis. O coeficiente de correlação de Spearman foi aplicado para determinar a relação entre o gasto com alimentação/1.000 kcal e o consumo de cada micronutriente. $\mathrm{O}$ valor amostral diferiu em cada análise de acordo com a disponibilidade dos dados. Considerou-se em $5 \%$ o nível de rejeição da hipótese de nulidade $(p<0,05)$. Os dados foram inseridos no programa Epi-Info, versão 6.4, com dupla digitação dos dados e posterior validate. As análises foram realizadas utilizando o programa SPSS, versão 11.0.

O projeto de pesquisa foi avaliado e aprovado pelo Comitê de Ética e Pesquisa da UFCSPA.

\section{Resultados}

O estudo foi realizado com 346 crianças com idade de 3 a 4 anos. Oito crianças, do total de 354 recrutadas na mesma faixa etária, foram excluídas das análises porque os responsáveis não estavam com as crianças no dia anterior, o que impossibilitava a realização do recordatório alimentar de 24 horas, ou porque estes não continham as quantidades ou preparações especificadas para a realização dos cálculos. Nas análises envolvendo renda familiar mensal e excesso de peso, 12 e nove crianças, respectivamente, não foram incluídas devido à perda destes dados. Em relação às análises envolvendo a ingestão dos micronutrientes ferro e vitaminas A e C, uma criança foi excluída por apresentar valores extremos.

As análises para avaliar o perfil socioeconômico da população do estudo utilizando o valor do salário-mínimo da época da coleta de dados ( $R \$ 374,67)$ mostraram que $51,5 \%$ das famílias apresentaram renda familiar mensal per capita inferior a 0,5 salário-mínimo. A porcentagem de mães com escolaridade inferior a 8 anos foi de $56,1 \%$, enquanto que a prevalência de famílias com mais de seis pessoas no domicílio e mais de dois filhos foi de 20,2 e $34,2 \%$, respectivamente.

A média dos gastos com a alimentação das crianças, estimada para 30 dias, foi de $R \$ 100,17 \pm 34,1$ e, ajustando para $1.000 \mathrm{kcal}$, a média foi de $\mathrm{R} \$ 66,34 \pm 15,89$. Os resultados envolvendo renda familiar mensal, utilizando os pontos de corte de acordo com os tercis de renda, mostraram que a média de gastos com alimentação/1.000 kcal das crianças foi mais elevada naquelas com maior renda familiar, como é mostrado na Tabela 1.

Em relação ao consumo de alimentos ADA e ADG, as análises mostraram que não houve diferença estatisticamente significativa na média de gastos com alimentação/1.000 kcal entre as crianças que consumiram diferentes quantidades desses alimentos. O gasto com a alimentação não foi diferente entre as crianças com ou sem risco para excesso de peso. Por outro lado, as análises em relação à qualidade nutricional mostraram correlação positiva entre a média de gastos com alimentação/1.000 kcal e a ingestão de ferro, zinco e vitaminas A e $C$ pelas crianças. A ingestão de todos os micronutrientes foi ajustada para $1.000 \mathrm{kcal}$, e as correlações estão apresentadas na Figura 1.

Tabela 1 - Média de gastos com alimentação/1.000 kcal estimada para 30 dias em relação a renda familiar total, variáveis dietéticas e excesso de peso em crianças de 3 a 4 anos de idade

\begin{tabular}{|c|c|c|c|}
\hline & $\mathbf{n}$ & Média de gasto/1.000 kcal (R\$) & $\mathbf{p}$ \\
\hline \multicolumn{4}{|c|}{ Renda familiar mensal total ( $R \$)$} \\
\hline$<600$ & 110 & $62,50 \pm 14,0$ & \\
\hline $600-950$ & 115 & $66,39 \pm 16,3$ & \\
\hline$>950$ & 109 & $70,42 \pm 16,5$ & $<0,001$ \\
\hline \multicolumn{4}{|l|}{ Calorias de ADA } \\
\hline$\leq 150 \mathrm{kcal}$ & 320 & $66,37 \pm 15,98$ & \\
\hline$>150 \mathrm{kcal}$ & 26 & $65,96 \pm 15,07$ & 0,894 \\
\hline \multicolumn{4}{|l|}{ Calorias de ADG } \\
\hline$\leq 150 \mathrm{kcal}$ & 242 & $66,37 \pm 16,59$ & \\
\hline$>150 \mathrm{kcal}$ & 104 & $66,29 \pm 14,19$ & 0,964 \\
\hline \multicolumn{4}{|c|}{ Risco para excesso de peso } \\
\hline Não ( $\leq 1$ escore $z$ ) & 266 & $65,93 \pm 14,55$ & \\
\hline $\operatorname{Sim}(>1$ escore $z)$ & 71 & $68,59 \pm 20,17$ & 0,208 \\
\hline
\end{tabular}

ADA = alimentos com alta densidade de açúcar (refrigerante, gelatina, bala e suco artificial); ADG = alimentos com alta densidade de gordura (salgadinho, bolacha recheada, embutidos, frituras e chocolate). 

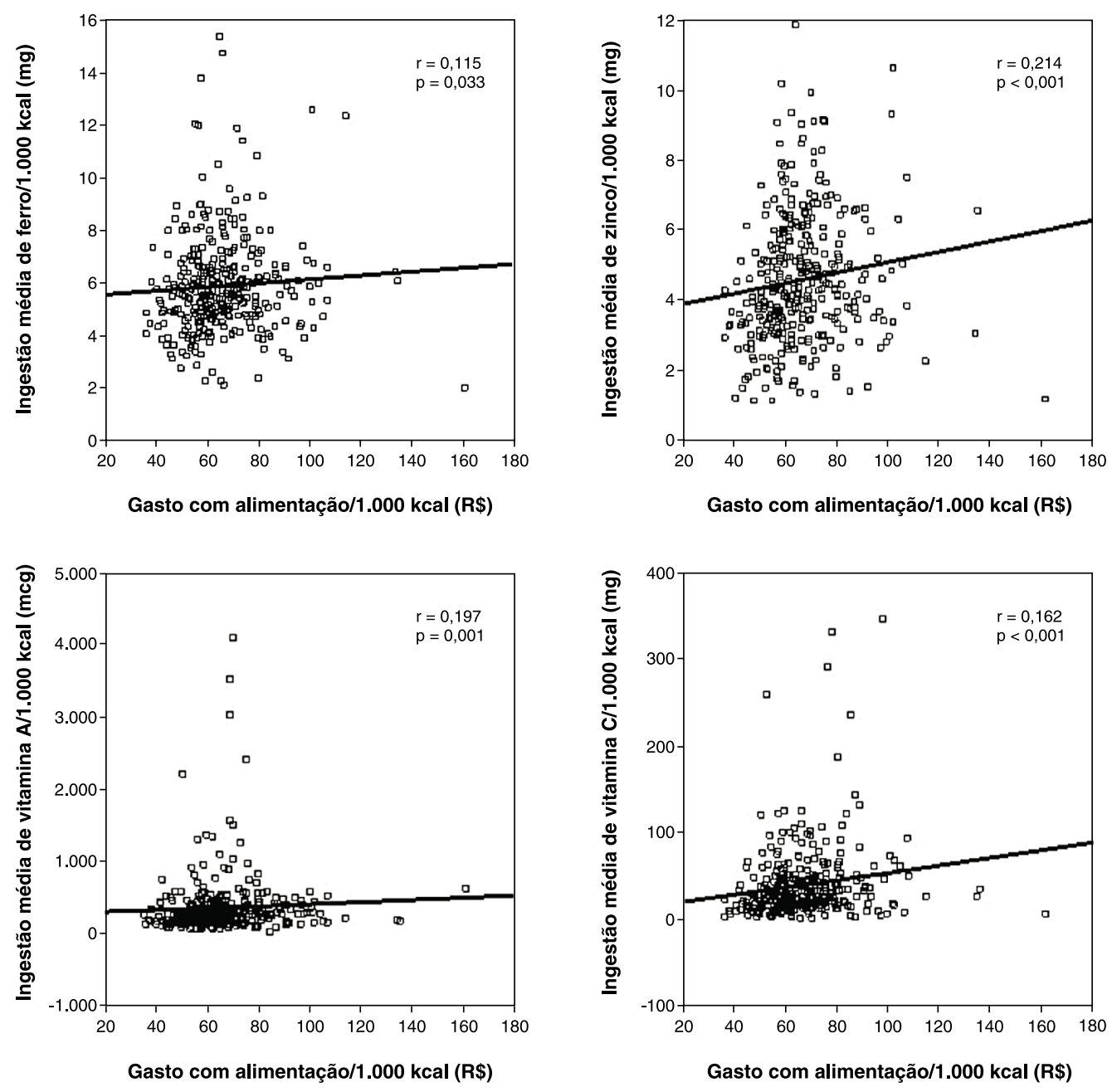

Figura 1 - Correlações da ingestão de micronutrientes ajustada para $1.000 \mathrm{kcal}$

\section{Discussão}

Os resultados deste estudo mostraram que os gastos com a alimentação das crianças, estimados para um mês, corresponderam a $27 \%$ do salário-mínimo ${ }^{12}$. Considerando que $51,5 \%$ das famílias apresentaram renda per capita inferior a 0,5 salário-mínimo e que os gastos com a alimentação de uma criança corresponderam em média a $60 \%$ do valor da cesta básica do Rio Grande do Sul no ano da coleta de dados $^{12}$, resulta evidente a importância de considerar os gastos com a alimentação nas orientações de promoção à saúde e prevenção de carências nutricionais e obesidade, principalmente nas populações de baixa condição socioeconômica. Neste estudo, observou-se aumento da aquisição de alimentos de melhor qualidade de acordo com a maior renda familiar. Além disso, a qualidade nutricional, avaliada pela ingestão de ferro, zinco e vitaminas A e C, mostrou correlação positiva com os gastos com alimentação/1.000 kcal. Esses micronutrientes foram selecionados devido a sua importância na saúde e crescimento de crianças menores de 5 anos. Esses resultados concordam com estudos que confirmaram a relação direta entre qualidade e gastos com a alimentação6,13.

Nesse estudo, o maior consumo de energia fornecida por alimentos com elevado percentual de açúcar e gordura não foi associado com os gastos com a alimentação, sugerindo facilidade para o consumo excessivo desses alimentos energéticos ${ }^{2}$ e o desenvolvimento de obesidade ${ }^{14,15}$ mesmo em condições econômicas limitadas ${ }^{16,17}$. Apesar de este estudo não ter encontrado associação entre excesso de peso e menor gasto com a alimentação, acredita-se que o período de exposição em função da idade das crianças tenha sido insuficiente para influenciar no ganho de peso excessivo. A formação dos hábitos alimentares inicia-se muito cedo, por volta dos 2 ou 3 anos de idade ${ }^{18}$, e em ambientes nos quais a alimentação é caracterizada por excesso de gordura e açúcar, alimentos mais palatáveis que verduras e legumes ${ }^{19}$ e que não influenciam no aumento dos gastos com a alimentação, o desenvolvimento da obesidade é mais prevalente 20,21 . Como agravante da situação nutricional inadequada, foi demonstrado que famílias de baixa renda e mães com menor esco- 
laridade oferecem alimentos ricos em gordura e açúcar com mais frequência a seus filhos ${ }^{22}$. Essas práticas alimentares caracterizadas por ingestão insuficiente de micronutrientes e excessiva de alimentos altamente energéticos podem contribuir para explicar em parte a alta prevalência de anemia23, hipovitaminose $\mathrm{A}^{24}$ e carência de outros micronutrientes, além da obesidade entre pré-escolares no Brasil 25 .

Publicações anteriores ao estudo BRATSA I ${ }^{26}$ mostraram que $75 \%$ das mães de crianças de baixo nível socioeconômico ofertaram vários tipos de guloseimas aos 12 meses de idade da criança. A implementação do programa "Dez passos da alimentação saudável: guia alimentar para crianças menores de dois anos" do Ministério da Saúde, por meio de orientações nutricionais às mães, foi efetiva em reduzir o consumo concomitante de alimentos de baixo valor nutricional, considerando que, no grupo-controle, o percentual de mães que ofereceram vários alimentos inadequados às crianças foi de $44,8 \%$, comparado com $27,2 \%$ das crianças do grupo-intervenção. Assim, é possível modificar positivamente as práticas alimentares de crianças sem que sejam necessárias grandes mudanças socioeconômicas; portanto, esse programa nacional sobre a alimentação saudável de crianças menores de 2 anos deve ser incorporado na rotina do Sistema Único de Saúde. Além disso, a agenda nacional de combate às deficiências nutricionais e à obesidade na infância devem urgentemente implementar as estratégias que garantam o acesso aos alimentos de qualidade e que sejam economicamente convenientes para esse grupo populacional, com vistas à prevenção do retardo de crescimento 27 , de prejuízo no desenvolvimento cognitivo ${ }^{24}$ e de desenvolvimento de obesidade futura ${ }^{14}$.

Os resultados deste estudo permitem concluir que o consumo de alimentos de alta densidade energética, ricos em açúcar e gordura, não está associado aos gastos com a alimentação, mas a qualidade nutricional de crianças de 3 a 4 anos, avaliada pela ingestão de micronutrientes necessários para o crescimento e desenvolvimento, mostrou estar correlacionada aos maiores gastos com a alimentação em grupos populacionais de baixo poder aquisitivo.

\section{Referências}

1. Levy-Costa RB, Sichieri R, Pontes NS, Monteiro CA. Disponibilidade domiciliar de alimentos no Brasil: distribuição e evolução (19742003). Rev Saude Publ. 2005;39:530-40.

2. Darmon N, Drewnoswski A. Does social class predict diet quality? Am J Clin Nutr. 2008;87:1107-17.

3. Monsivais $P$, Drewnowski $A$. The rising cost of low-energy-density foods. J Am Diet Assoc. 2007;107:2071-6.

4. Drewnoswski A, Specter SE. Poverty and Obesity: the role of energy density and energy costs. Am J Clin Nutr. 2004;79:6-16.

5. Townsend MS, Aaron GJ, Monsivais P, Keim NL, Drewnowski A. Less-energy-dense diets of low-income women in California are associated with higher energy-adjusted diet costs. Am J Clin Nutr. 2009;89:1220-6.

6. Maillot M, Darmon N, Darmon M, Lafay L, Drewnowski A. Nutrientdense food groups have high energy costs: an econometric approach to nutrient profiling. J Nutr. 2007;137:1815-20.

7. Ministério da Saúde. Dez passos da alimentação saudável: guia alimentar para crianças menores de dois anos. Brasília (DF): Ministério da Saúde/Organização Pan-Americana da Saúde; 2002.
8. World Health Organization (WHO). [website] Anthro Manual. 2005. Beta version: software for assessing growth and development of the world's children. Geneva: WHO; 2006. http://www.who. int/childgrowth/software/en/. Acesso: 17/02/2006.

9. Núcleo de Estudos e Pesquisas em Alimentação. Universidade Estadual de Campinas [NEPA/Unicamp]. Tabela Brasileira de Composição de Alimentos [TACO]: versão 2. São Paulo: NEPA/ UNICAMP; 2006.

10. Philippi ST. Tabela de composição de alimentos: suporte para decisão nutricional. São Paulo: Metha; 2002.

11. Institute of Medicine of the National Academies. Dietary reference intakes: energy, carbohydrates, fiber, fat, fatty acids, cholesterol, protein and amino acids. Washington: National Academies Press; 2002.

12. Departamento Intersindical de Estatística e Estudos Socioeconômicos. [website] Pesquisa Nacional da Cesta Básica. http//www.dieese.org.br. Acesso: 22/05/2008.

13. Beydoun MA, Powell LM, Wang Y. The association of fast food, fruit and vegetable prices with dietary intakes among US adults: is there modification by family income? Soc Sci Med. 2008;66:2218-29.

14. Nicklas TA, Yang SJ, Baranowski T, Zakeri I, Berenson G. Eating patterns and obesity in children. The Bogalusa Heart Study. Am J Prev Med. 2003;25:9-16.

15. St-Onge MP, Keller KL, Heymsfield SB. Changes in childhood food consumption patterns: a cause for concern in light of increasing body weights. Am J Clin Nutr. 2003;78:1068-73.

16. Monteiro CA, Conde WL, Lu B, Popkin BM. Obesity and inequities in health in the developing world. Int J Obes Relat Metab Disord. 2004;28:1181-6.

17. Drewnowski A, Darmon N. Food choices and diet costs: an economic analysis. J Nutr. 2005;135:900-4

18. Skinner JD, Carruth BR, Wendy B, Ziegler PJ. Children's food preferences: a longitudinal analysis. J Am Diet Assoc. 2002;102:1638-47.

19. Levine AS, Kotz CM, Gosnell BA. Sugars and fats: the neurobiology of preference. J Nutr. 2003;133:831S-4S.

20. Swinburn BA, Caterson I, Seidell JC, James WP. Diet, nutrition and the prevention of excess weight gain and obesity. Public Health Nutr. 2004;7:123-46.

21. Stubbs RJ, Whybrow S. Energy density, diet composition and palatability: influences on overall food energy intake in humans. Phisiol Behav. 2004;81:755-64.

22. Cooke LJ, Wardle J, Gibson EL, Sapochnik M, Sheiham A, Lawson M. Demographic, familial and trait predictors of fruit and vegetable consumption by pre-school children. Public Health Nutr. 2004; 7:295-302.

23. Vieira AC, Diniz AS, Cabral PC, Oliveira RS, Lóla MM, Silva SM, et al. Nutritional assessment of iron status and anemia in children under 5 years old at public daycare centers. J Pediatr (Rio J). 2007;83:370-6.

24. Diniz AS, Santos LM. Hipovitaminose A e xeroftalmia. J Pediatr (Rio J). 2000;76:311-22.

25. Coutinho JG, Gentil PC, Toral N. A desnutrição e obesidade no Brasil: o enfrentamento com base na agenda única da nutrição. Cad Saude Publ. 2008;24:332-40.

26. Vitolo MR, Bortolini GA, Feldens CA, Drachler ML. Impactos da implementação dos dez passos da alimentação saudável para crianças: ensaio de campo randomizado. Cad Saude Publica. 2005;21:1448-57.

27. Silva AP, Vitolo MR, Zara LF, Castro CF. Effects of zinc supplementation on 1 - to 5-year old children. J Pediatr (Rio J). 2006;82:227-31.

Correspondência:

Fernanda Rauber

Rua Sarmento Leite, 245

CEP 90050-170 - Porto Alegre, RS

Tel.: (51) 3303.8798

Fax: (51) 3303.8798

E-mail: rauber.fernanda@gmail.com 